\title{
GREEN'S FUNCTIONS FOR MULTIPLY CONNECTED DOMAINS VIA CONFORMAL MAPPING *
}

\author{
MARK EMBREE ${ }^{\dagger}$ AND LLOYD N. TREFETHEN $\ddagger$
}

\begin{abstract}
A method is described for the computation of the Green's function in the complex plane corresponding to a set of $K$ symmetrically placed polygons along the real axis. An important special case is a set of $K$ real intervals. The method is based on a Schwarz-Christoffel conformal map of the part of the upper half-plane exterior to the problem domain onto a semi-infinite strip whose end contains $K-1$ slits. From the Green's function one can obtain a great deal of information about polynomial approximations, with applications in digital filters and matrix iterations. By making the end of the strip jagged, the method can be generalized to weighted Green's functions and weighted approximations.
\end{abstract}

Key words. Green's function, conformal mapping, Schwarz-Christoffel formula, polynomial approximation, potential theory, Chebyshev polynomial, digital filter, Krylov subspace iteration

AMS subject classifications. 30E10, 31A99, 41A10, 65F10

1. Introduction. Green's functions in the complex plane are basic tools for the analysis of real and complex polynomial approximations [10,21,24,30,32], which are of central importance in the fields of digital signal processing $[16,17,19]$ and matrix iterations $[5,6,11,20,28]$. The aim of this article is to show that when the domain of approximation is a collection of real intervals, or more generally symmetric polygons along the real axis, the Green's function can be computed to high accuracy by SchwarzChristoffel conformal mapping. The computation of Schwarz-Christoffel maps has become routine in recent years with the introduction of Driscoll's MATLAB ${ }^{\circledR}$ SchwarzChristoffel Toolbox [4], a descendant of the second author's Fortran package SCPACK [26].

The Green's function for a single interval can be obtained by a Joukowsky conformal map, and related polynomial approximation problems were solved by Chebyshev in the 1850s [3]. For two disjoint intervals, the Green's function can be expressed using elliptic functions, and approximation problems were investigated by Akhiezer in the 1930s [2]. For $K>2$ intervals, the Green's function can be derived from a more general Schwarz-Christoffel conformal map, and the formulas that result were stated in a landmark article by Widom in 1969 [32]. Polynomial approximations can be readily computed in this case by the Remes algorithm, which was adapted for digital filtering by Parks and McClellan $[3,18]$.

By a second conformal map, these ideas for intervals can be transplanted to the more general problem of the Green's function for the region exterior to a string of symmetric domains along the real axis ([32], p. 230). The conformal maps in question can usually not be determined analytically, however, and even for the case of intervals on the real axis, the formula for the Green's function requires numerical integration. Here, for the case in which the domains are polygonal and thus can be reduced to

*Received by the editors XXX x, 19xx; accepted by the editors XXX x, 19xx. This work was supported by NSF Grant DMS-9500975CS (US) and EPSRC Grant GR/M12414 (UK).

† Oxford University Computing Laboratory, Wolfson Building, Parks Road, Oxford, UK (embree @comlab.ox.ac.uk).

$\ddagger$ Oxford University Computing Laboratory, Wolfson Building, Parks Road, Oxford, UK (LNT@ comlab.ox.ac.uk). 
intervals by a Schwarz-Christoffel map, we carry out the computations to put these ideas into practice.

This article originated from discussions with Steve Mitchell of Cornell University, who is writing a dissertation on applications of these ideas to the design of multirate filters [15], and we are grateful to him for many suggestions. The contributions of Jianhong Shen and Gilbert Strang at MIT were also a crucial help to us. Shen and Strang have studied the accuracy of lowpass digital filters $[22,23]$, and their asymptotic formulas are directly connected to these Schwarz-Christoffel methods. In addition we thank Toby Driscoll for his advice and assistance.

Our algorithm makes possible the computational realization of results in approximation theory going back to Faber, Szegö, Walsh, Widom, and Fuchs, among others. In particular, Walsh, Russell, and Fuchs obtained theorems concerning simultaneous approximation of distinct entire functions on disjoint sets in the complex plane $[8,9,30]$, which we illustrate here in Section 6. Wolfgang Fuchs was for many years a leading figure at Cornell University until his unfortunate death in 1997.

2. Description of the algorithm. Let $E$ be a compact subset of the complex plane consisting of $K$ disjoint polygons $P_{1}, \ldots, P_{K}$ numbered from left to right, with each polygon symmetric with respect to the real axis. Degenerate cases are permitted in which a portion of a polygon, or all of it, reduces to a line segment (but not to a point). The Green's function problem for $E$ is defined as follows:

Green's Function Problem. Find a real function $g$ defined in the region of the complex plane exterior to E satisfying

$$
\begin{aligned}
\Delta g(z) & =0 \text { for } z \notin E, \\
g(z) & \rightarrow 0 \text { for } z \rightarrow E, \\
g(z) & \sim \log |z| \text { for } z \rightarrow \infty .
\end{aligned}
$$

In (1a), $\Delta$ denotes the Laplacian operator, and thus $g$ is harmonic throughout the complex plane exterior to the polygons $P_{j}$. Standard results of potential theory ensure that there exists a unique function $g$ satisfying these conditions $[12,13,29,32]$.

The solution to (1) can be constructed by conformal mapping. What makes this possible is that the problem is symmetric with respect to the real axis, so it is enough to find $g(z)$ for the part of the upper half-plane $\operatorname{Im} z \geq 0$ exterior to $E$; the solution in the lower half-plane is then obtained by reflection (the Schwarz reflection principle). This half-planar region is bounded by the upper halves of the polygons $P_{j}$ and by the intervals along the real axis that separate the polygons, where the appropriate boundary condition for $g$, by symmetry, is the Neumann condition $d g / d n=0$.

Restricting the map to the upper half-plane makes the domain simply-connected, suggesting the following conformal mapping problem.

Conformal Mapping Problem. Find an analytic function $f$ that maps the portion of the upper half-plane exterior to $E$ (Fig. 1a) conformally onto a semi-infinite slit strip (Fig. 1c). Only the vertices $f\left(s_{1}\right)=$ $\pi i, f\left(T_{K}\right)=0$, and $f(\infty)=\infty$ are prescribed. The remaining vertices, and hence the lengths and heights of the slits, are not specified.

Once this mapping problem is solved, the function $g$ defined by

$$
g(z)=\operatorname{Re}(f(z))
$$


(a)



(b)


(c)

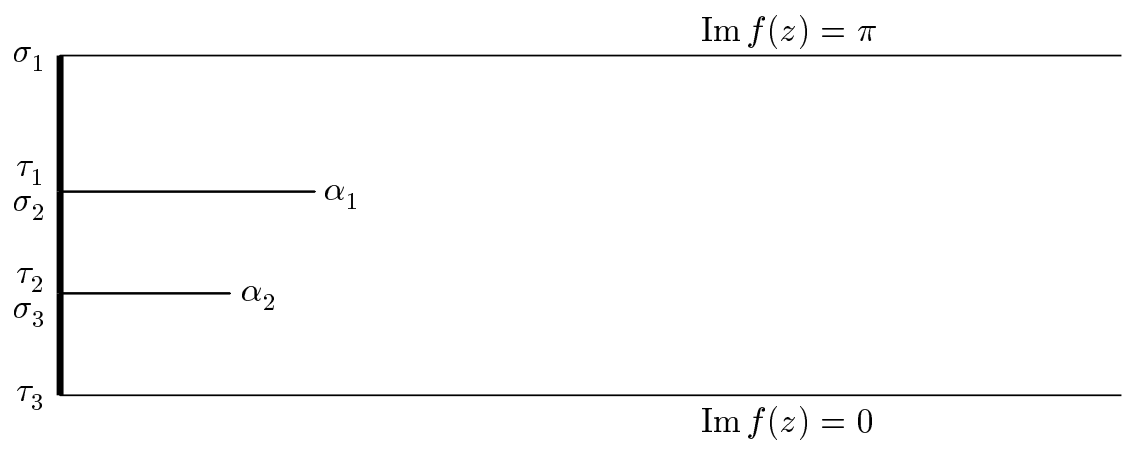

Figure 1. Determination of the Green's function $g(z)$ by a composition of two conformal maps, $g(z)=\operatorname{Re} f(z)=\operatorname{Re} f_{2}\left(f_{1}(z)\right)$. (a) The problem domain is restricted to the part of the upper half-plane exterior to the polygons $P_{j}$. (b) The first Schwarz-Christoffel map $f_{1}$ takes this problem domain onto the upper half-plane itself. (c) The second Schwarz-Christoffel map $f_{2}$ takes the upper half-plane to a slit semiinfinite strip. The interval $\left[s_{j}, t_{j}\right]$ maps to a vertical boundary segment $\left[\sigma_{j}, \tau_{j}\right]$ with $\operatorname{Re}\left[\sigma_{j}, \tau_{j}\right]=0$. The gaps along the real axis between the intervals $\left[s_{j}, t_{j}\right]$ map to horizontal slits, and the semi-infinite intervals $\left(-\infty, s_{1}\right)$ and $\left(t_{n}, \infty\right)$ map to semi-infinite horizontal lines with imaginary parts $\pi$ and 0 , respectively. Only the real parts of the left endpoints of the slits are prescribed; the imaginary parts and the right endpoints $\alpha_{j}$, as well as their pre-images $a_{j}$, are determined as part of the calculation.

is the Green's function (1) for values of $z$ in the upper half-plane. To see this we note that $g$ satisfies (1a) because the real part of an analytic function is harmonic, it satisfies (1b) because of the form of the slit strip, and it satisfies (1c) because the half-strip has height $\pi$. The existence and uniqueness of a solution to the Conformal Mapping Problem can be derived from standard theory of conformal mapping [12] or as a consequence of the corresponding facts for the Green's Function Problem. 




Figure 2. Composition of a third conformal map, the complex exponential, transplants the slit strip to the exterior of a disk with radial spikes in the upper half-plane. Reflection in the real axis completes the map of the problem domain of Fig. 1a, yielding a function $w=\Phi(z)$ such that $g(z)=\log |\Phi(z)|$.

The function $f(z)$ is a conformal map from one polygon to another, and as such, it can be represented by Schwarz-Christoffel formulas, an idea going back to Schwarz and independently Christoffel around 1869. Figure 1 shows how $f$ can be constructed as the composition of two Schwarz-Christoffel maps. The first one maps the problem domain in the upper half-plane to the upper half-plane, with the upper half of the boundary of the polygon $P_{j}$ going to the interval $\left[s_{j}, t_{j}\right]$. This mapping problem is a standard one, for which a parameter problem must be solved to determine accessory parameters in the Schwarz-Christoffel formula; see $[4,12,26]$. By the second SchwarzChristoffel map, the upper half-plane is then mapped to the slit strip. This is a Schwarz-Christoffel problem in the reverse, more trivial direction, with only a linear parameter problem to be solved to impose the condition that the upper and lower sides of each slit have equal length. Details can be found in [23] and [32]. A related linear Schwarz-Christoffel problem involving slits in the complex plane is implicit in [14].

By composing a third conformal map with the first two, we obtain a picture that is even more revealing than Fig. 1. Figure 2 depicts the image of the slit strip under the complex exponential: $w=\Phi(z)=\exp \left(f_{2}\left(f_{1}(z)\right)\right)$. The vertical segments now map onto arcs of the upper half of the unit circle, the slits map onto radial spikes protruding from that circle, and the infinite horizontal lines map to the portion of the real axis exterior to the circle. The real axis is shown dashed, because we immediately reflect across it to get a complete picture.

By the composition $\Phi(z)$ of three conformal maps, we have transplanted the $K$-connected exterior of the region $E$ of Fig. 1a to the simply-connected exterior of the spiked unit disk of Fig. 2. (These connectivities are defined with respect to the Riemann sphere or the extended complex plane $\mathbf{C} \cup\{\infty\}$.) The Green's function for $E$ is given by the extraordinarily simple formula

$$
g(z)=\log |\Phi(z)|
$$

Have we really mapped a $K$-connected region conformally onto a simply-connected region? No, this is is not possible, and to resolve what looks like a contradiction we must think more carefully about reflections. Suppose in Fig. 1a we think of 
the finite dashed intervals as branch cuts not to be crossed, and reflect only across the semi-infinite dashed intervals at the ends. Then the complement of $E$ becomes simply-connected, and we have indeed constructed a conformal map onto the simplyconnected region of Fig. 2. However, the Schwarz reflection principle permits reflection across arbitrary straight lines or circular arcs. There is no reason why one should exclude the finite intervals in Fig. 1a as candidates, which would correspond in the $w$-plane to reflection in the protruding spikes of Fig. 2. When such reflections are allowed, $\Phi(z)$ becomes a multi-valued function whose values depend on paths in the complex plane - or equivalently, a single-valued conformal map of Riemann surfaces. Even under arbitrary reflections with arbitrary multi-valuedness, fortunately, equation (3) remains valid, since all reflections preserve the absolute value $|\Phi(z)|$ and $g(z)$ depends only on this absolute value. Therefore, for the purpose of calculating Green's functions, we escape the topological subtleties of the conformal mapping problem.

The phenomenon of multivaluedness is a familiar one in complex analysis. An analysis of the multivalued function $\Phi(z)$ is the basis of Widom's approximation theoretic results in [32], and earlier discussions of the same function can be found, for example, in [30] and [31].

3. Computed example; electrostatic interpretation. Our first computed example is presented in detail to illustrate our methods. The region $E$ of Fig. 3(a) has $K=2$ polygons, a red hexagon and a green square. (The hexagon is defined by coordinates $-6.5,-5 \pm 1.5 i,-5.75 \pm 2.25 i,-8$ and the square by coordinates 9.5 , $8.75 \pm 0.75 i, 8$.) In Fig. $3(\mathrm{~b})$, three subsets of the real axis have been introduced, blue and turquoise and magenta, to complete the boundary of the half-planar region. Plots (c) and (d) show the conformal images of this region as a slit strip and the exterior of the disk with a spike. The color coding is maintained to indicate which boundary segments map to which.

All of these computations, like those in our later examples, have been carried out with the high accuracy that comes cheaply in Schwarz-Christoffel mapping [26]. Thus our figures can be regarded as exact for plotting purposes. For the sake of those who may wish to duplicate some of these computations, in the sections below we report occasional numbers, which are believed in each case to be correct to all digits listed.

Green's functions have a physical interpretation in terms of two-dimensional electric charge distributions, that is, cross-sections of infinite parallel line charge distributions in three dimensions. In Fig. 3(d), the equilibrium distribution of one (negative) unit of charge along the unit circle is the uniform distribution, which generates the associated potential $g(w)=\log |w|$. By conformal transplantation under the map $z=\Phi^{-1}(w)$, this maps to a non-uniform distribution along the boundaries of the polygons $P_{j}$ in the $z$-plane. This nonuniform charge distribution on the polygons $P_{j}$ is precisely the minimal-energy, equilibrium charge distribution for these sets. It is the charge distribution that would be achieved if each polygon were an electrical conductor connected to the other polygons by wires in another dimension so as to put them all at the same voltage. Mathematically, the charge distribution is distinguished by the special property that it generates the potential $g(z)$ with constant value on the boundaries of the polygons.

4. Asymptotic convergence factor, harmonic measure, and capacity. Every geometrical detail of Fig. 3 has a mathematical interpretation for the Green's function problem, which becomes a physical interpretation if we think in terms of equilibrium charge distributions. We now describe several items that are particularly important. 

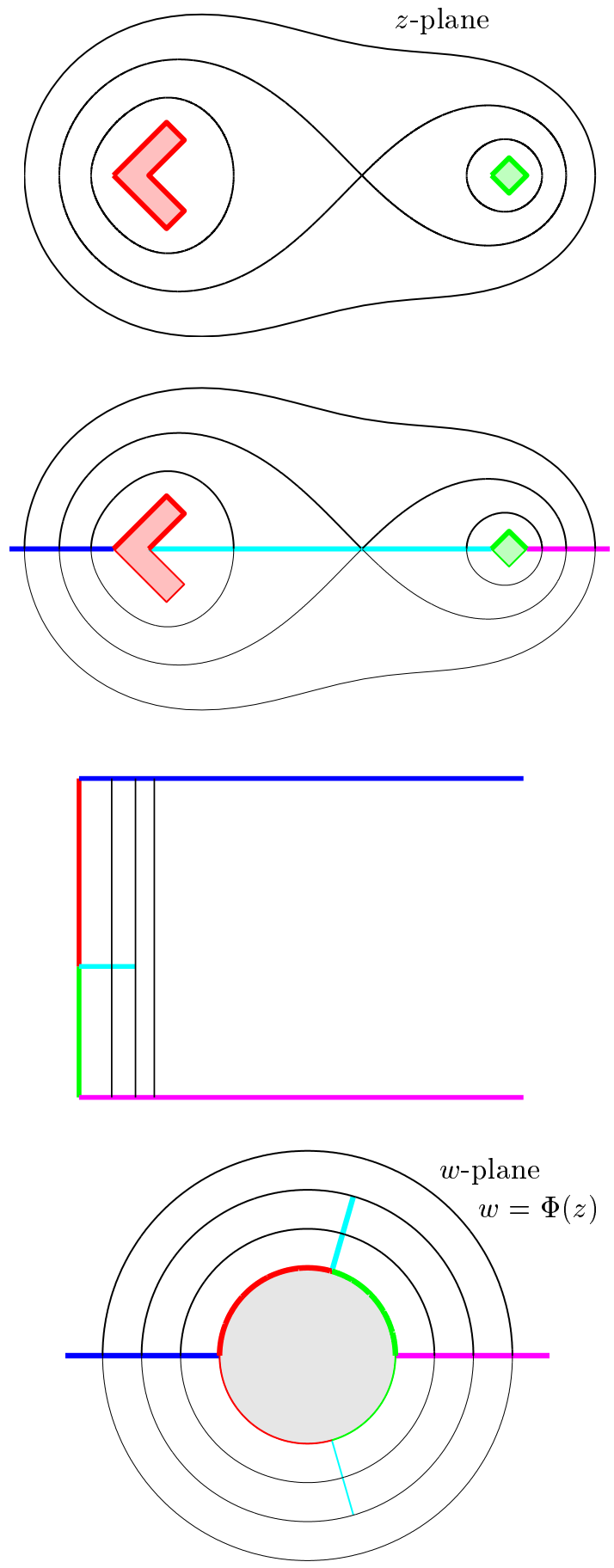

(a) Problem domain, showing the computed critical level curve $g(z)=g_{c}$ as well as one lower and one higher level curve.

(b) To obtain these results, first the real axis is drawn in as an artificial boundary. Heavy lines mark the boundary of the new simplyconnected problem domain.

(c) The half-planar region is then transplanted by a composition of two Schwarz-Christoffel maps to a slit semi-infinite strip. The real interval between the polygons (turquoise) maps to a horizontal slit whose coordinates are determined as part of the solution. Vertical lines in the strip correspond to level curves of the Green's function of the original problem.

(d) Finally, the exponential function maps the strip to the upper half of the exterior of the unit disk. The slit becomes a protruding turquoise spike. Here the Green's function is $\log |w|$, with concentric circles as level curves. Reflection extends the circles to the lower halfplane, and following the maps in reverse produces the curves of (a).

Figure 3. Color-coded computed illustration of our algorithm for an example with $K=2$ polygons. The blue, red, turquoise, green, and magenta boundary segments in the various domains correspond under conformal maps. Fainter lines distinguish function values obtained by reflection. 
Critical point, potential, and level curve. For sufficiently small $\epsilon>0$, the region of $\mathbf{C} \backslash E$ where $g(z)<\epsilon$ consists of $K$ disjoint open sets surrounding the polygons $P_{j}$. At some value $g_{c}$, two of these sets first coalesce at a point $z_{c} \in \mathbf{R}$, which will be a saddle point of $g(z)$, i.e., a point where the gradient of $g(z)$ and also the complex derivative $\Phi^{\prime}(z)$ are zero [30]. We call $z_{c}$ the critical point, $g_{c}$ the critical potential, and $\left\{z \in \mathbf{C}: g(z)=g_{c}\right\}$ the critical level curve. (We speak as if $z_{c}$ is a single point and just two sets coalesce there, which is the generic situation, but in special cases there may be more than one critical point and more than two coalescing regions, as in Fig. 7 below.)

These critical quantities can be immediately obtained from the geometry of our conformal mapping problem. Let $w_{c}$ denote the endpoint of the shortest protruding spike as in Figs. 2 or $3(\mathrm{~d})$. Then $z_{c}=\Phi^{-1}\left(w_{c}\right)\left(=f_{1}^{-1}\left(a_{j_{c}}\right)\right.$, where $j_{c}$ is the index of the critical point $a_{j}$ as in Figure 1), $g_{c}=\log \left(\left|w_{c}\right|\right)$, and the critical level curve is the pre-image under $\Phi$ of the circle $|w|=\left|w_{c}\right|$. For the example of Fig. 3, $z_{c}=2.517348$, $g_{c}=0.634942$, and the critical level curve is plotted in Fig. 3(a).

Asymptotic convergence factor. In applications to polynomial approximation, as described in Section 6, the absolute value of the end of the shortest spike is of particular interest. With the same notation as above, we define the asymptotic convergence factor associated with $g(z)$ by

$$
\rho=\left|w_{c}\right|^{-1}=\exp \left(-g_{c}\right)
$$

For the example of Fig. 3, $\rho=0.529966$.

Note that $g_{c}$ and $\rho$ depend on the shape of the domain $E$, but not on its scale. Doubling the sizes of the polygons and the distances between them, for example, does not change these quantities. They are also invariant with respect to translation of the set $E$ in the complex plane.

Harmonic measure. Another scale-independent quantity is the proportion $\mu_{j}$ of the total charge on each polygon $P_{j}$, which is known as the harmonic measure of $P_{j}$ (with respect to the point $z=\infty$ ) $[1,7,13]$. This quantity is equal to $\pi^{-1}$ times the distance between the appropriate two slits in the strip domain (or a slit and one of the semi-infinite boundary lines), or equivalently to $\pi^{-1}$ times the angle between two spikes (or a spike and the real axis) in the $w$-plane. In the notation of Fig. 1,

$$
\mu_{j}=\frac{\sigma_{j}-\tau_{j}}{\pi i}
$$

For the example of Fig. 3, the slit is at height $\operatorname{Im} \sigma_{2}=1.290334$, and dividing by $\pi$ shows that the proportion of charge on the green square is $\mu_{2}=0.410726$. The density of charge at particular points along the boundary is equal to $\pi^{-1}|d w / d z|=\pi^{-1}\left|\Phi^{\prime}(z)\right|$, a number that is easy to evaluate since the Schwarz-Christoffel formula expresses $\Phi(z)$ in terms of integrals. (This density can be used to define the harmonic measure of arbitrary measurable subsets of the boundary of $E$, not just of the boundary of $P_{j}$.)

Capacity. The capacity $C$ (= logarithmic capacity, also called the transfinite diameter) of a compact set $E \subset \mathbf{C}$ is a standard notion in complex analysis and approximation theory $[1,13]$. This scale-dependent number can be defined informally as the average distance between charges, in the geometric-mean sense, for an equilibrium charge density distribution on the boundary of $E$. Familiar special cases are $C=R$ for a disk of radius $R$ and $C=L / 4$ for an interval of length $L$. For a general domain $E, C$ is equal to the derivative $d z / d w$ evaluated at $z=w=\infty$, that is, $C=1 / \Phi^{\prime}(\infty)$. (Normally one would have absolute values, but for our problem $\Phi^{\prime}(\infty)$ is real and positive.) 
One way to compute $C$ is to note that $\Phi(z)$ is the composition of $f_{1}(z)$ and $\exp \left(f_{2}(z)\right)$, in the notation of Figs. 1 and 2 , and $f_{1}^{\prime}(\infty)$ is just the multiplicative constant of the first of our two Schwarz-Christoffel maps. Thus the crucial quantity to determine is the limit of $z / \exp \left(f_{2}(z)\right)$ as $z \rightarrow \infty$, whose logarithm is given by

$$
\begin{aligned}
\lim _{z \rightarrow \infty}\left(\log z-f_{2}(z)\right) & =\lim _{z \rightarrow \infty}\left[\int_{1}^{z} \zeta^{-1} d \zeta-\int_{t_{K}}^{z} f_{2}^{\prime}(\zeta) d \zeta\right] \\
& =\int_{t_{K}}^{\infty}\left(\frac{1}{z+1-t_{K}}-f_{2}^{\prime}(z)\right) d z
\end{aligned}
$$

since $f_{2}\left(t_{K}\right)=0$. This is a convergent integral of Schwarz-Christoffel type that can be evaluated accurately by numerical methods related to those of SCPACK and the SC Toolbox.

Alternatively, we have found that sufficient accuracy can be achieved without the explicit manipulation of integrals. Using the Schwarz-Christoffel maps, we calculate the quantities

$$
C(z)=\frac{z}{\Phi(z)}
$$

for a collection of values of $z$ such as $z=2^{j}, j=10, \ldots, 15$. The function $C(z)$ is analytic at $z=\infty$, and the capacity $C=C(\infty)$ can be obtained in a standard manner by Richardson extrapolation. For the example of Fig. 3, $C=4.082273$.

The ideas of this section can be spelled out more fully in formulas, generally integrals or double integrals involving the charge density distribution; see [13,21,29]. We omit these details here.

5. Further examples. Figures $4-7$ present computed examples with $K=2,3,4$ and 5 polygons. In each case, the critical level curve of $g(z)$ has been plotted together with three level curves outside the critical one. In the case of Fig. 6 , a fifth level curve has also been plotted that corresponds to the highest of the three saddle points of $g(z)$ for that problem. If the small square on the right in that figure were not present, then by symmetry, there would be two saddle points between the long quadrilaterals at the same value of $g(z)$. The square, however, breaks the symmetry, moving those saddle points to the slightly distinct levels $g(z)=3.491 \times 10^{-4}$ (shown) and $g(z)=$ $3.681 \times 10^{-4}$ (not shown).

Figure 7 may puzzle the reader. Why does the critical level curve self-intersect at four points, indicating four saddles at exactly the same level, even though there is no left-right symmetry in the figure? The answer is that the coordinates of the squares in this example have been adjusted to make this happen. The widths of the squares are $1,2,3,4$, and 5 , with the left-hand edges of the first two located at $x=0$ and $x=4$. This gave us a system of three nonlinear equations in three unknowns to solve for the locations of the remaining three left-hand edges that would achieve the uniform critical value. (This is an example of a generalized Schwarz-Christoffel parameter problem, in which geometric constraints from various domains are mixed [27].) The locations that satisfy the conditions are $10.948290,20.326250$, and 31.191359 , the critical potential value is $g_{c}=0.0698122$, and the capacity is $C=10.292969$.

6. Applications to polynomial approximation. Many uses of Green's functions pertain to problems of polynomial approximation. The basis of this connection is an elementary fact: if $p(z)=\prod\left(z-z_{j}\right)$, then $\log |p(z)|=\sum \log \left|z-z_{j}\right|$, and thus the size of a polynomial $p(z)$ is essentially the same as the value of the potential generated 


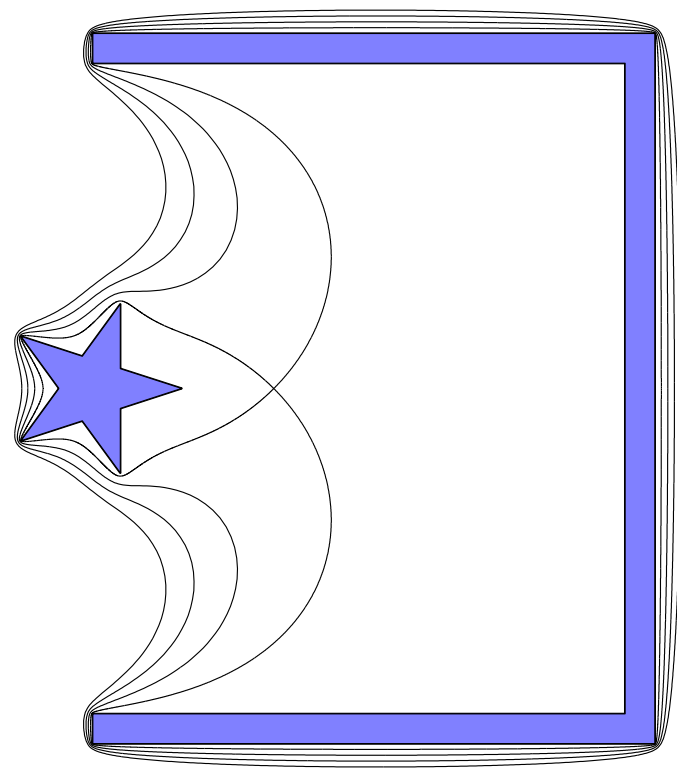

Figure 4. Green's function for a region defined by two polygons. This computation is identical in structure to that of Fig. 3.

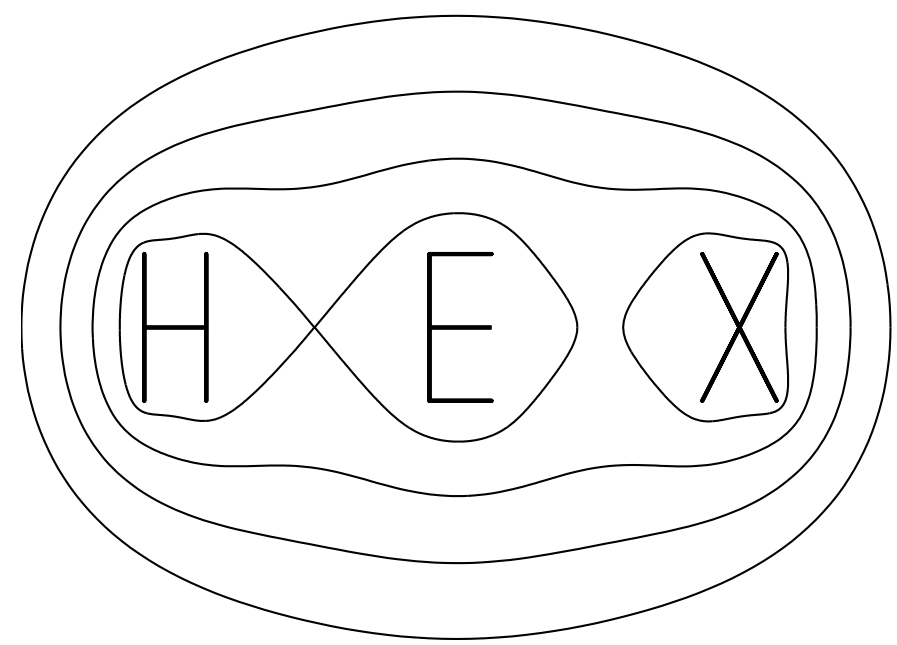

Figure 5. Green's function for a region defined by three degenerate polygons with empty interior. As it is exteriors that are conformally mapped, the degeneracy has no effect on the mathematical problem or the method of solution.

by "point charges" with potentials $\log \left|z-z_{j}\right|$ located at its roots $\left\{z_{j}\right\}$. In the limit as the number of roots and charges goes to $\infty$, one obtains a continuous problem such as (1). Generally speaking, the properties of optimal degree- $n$ polynomials for various approximation problems can typically be determined to leading order as $n \rightarrow \infty$ from the Green's function in the sense that we get the exponential factors right but not the algebraic ones. Numerous results in this vein are set forth in the treatise of Walsh 


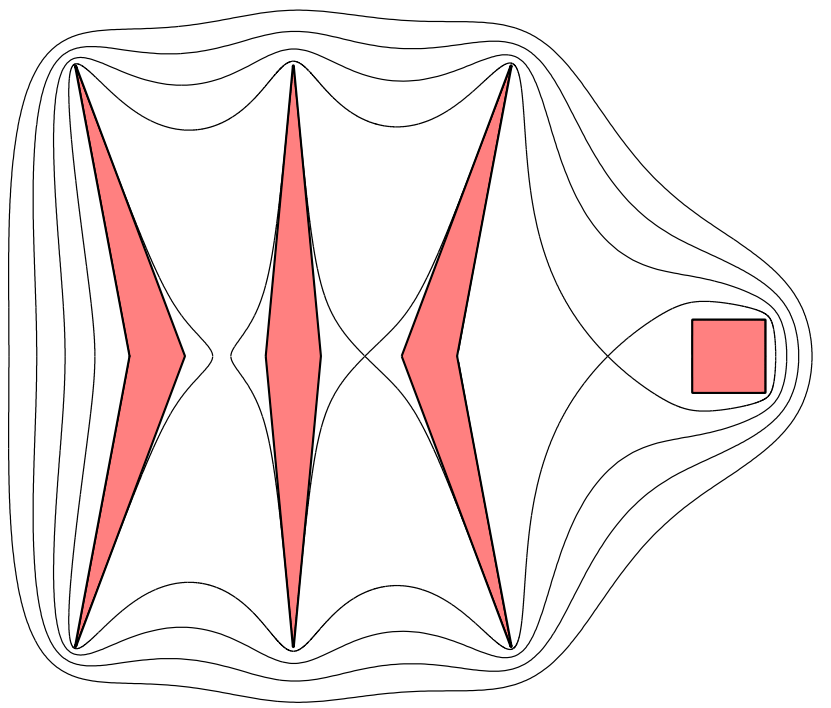

Figure 6. Green's function for a region defined by four polygons. The square on the side breaks the symmetry.

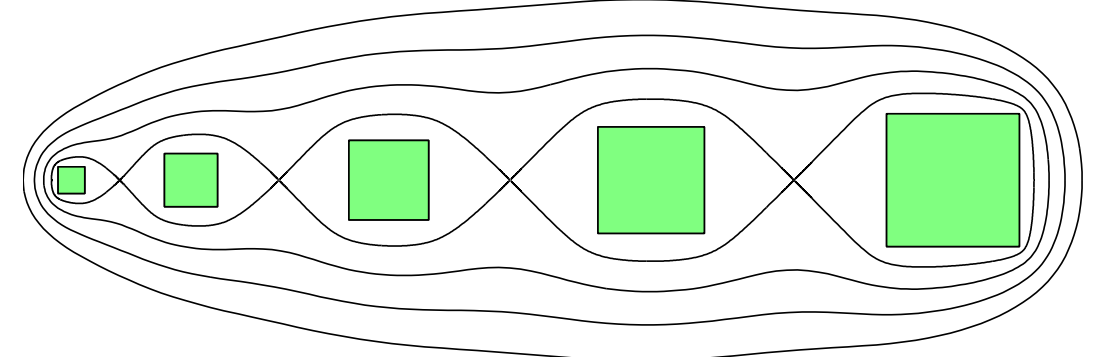

Figure 7. Green's function for a region defined by five polygons. The spacing of the squares has been adjusted to make all the critical points lie at the same value $g_{c}$.

[30].

Perhaps the simplest approximation topic one might consider is the Chebyshev polynomials $\left\{T_{n}\right\}$ associated with a compact set $E \subseteq \mathbf{C}$. For each $n, T_{n}$ is defined as the monic polynomial of degree $n$ that minimizes $\left\|T_{n}\right\|=\max _{z \in E}\left|T_{n}(z)\right|$. The following result indicates one of the connections between $T_{n}$ and the Green's function for $E$.

THEOREM 1. Let $E \subseteq \mathbf{C}$ be a compact set with capacity $C$. Then a unique Chebyshev polynomial $T_{n}$ exists for each $n \geq 0$, and

$$
\lim _{n \rightarrow \infty}\left\|T_{n}\right\|^{1 / n}=C \text {. }
$$

It follows from this theorem that the numerical methods of this paper enable us to determine the leading order behavior of Chebyshev polynomials for polygons symmetrically located on the real axis. For example, the $n$th Chebyshev polynomial of the five-square region of Fig. 7 has size approximately $(10.292969)^{n}$. Other related matters, such as generalized Faber polynomials [32], can also be pursued. 
Theorem 1 is due to Szegö [25], who extended earlier work of Fekete; a proof can be found for example in [29]. For the case in which $E$ is a smooth Jordan domain, Faber showed that in fact $\left\|T_{n}\right\| / C^{n} \rightarrow 1$ as $n \rightarrow \infty$. If $E$ consists of two intervals, Akhiezer showed that $\left\|T_{n}\right\| / C^{n}$ oscillates between two constants, and the starting point of the paper of Widom [32] is the generalization of this result to a broad class of sets $E$ with multiple components.

Instead of discussing Chebyshev polynomials further, we shall consider a different, related approximation problem investigated by Walsh, Russell, and Fuchs, among others $[8,9,30,31]$. Let $h_{1}, h_{2}, \ldots, h_{K}$ be entire functions, i.e., each $h_{j}$ is analytic throughout the complex plane, and to keep the formulations simple, assume that these functions are distinct. The following is a special case of the general complex Chebyshev approximation problem:

Polynomial Approximation Problem. Given n, find a polynomial $p_{n}$ of degree $n$ that minimizes the quantity

$$
E_{n}=\max _{1 \leq j \leq K} \max _{z \in P_{j}}\left|p_{n}(z)-h_{j}(z)\right|
$$

Note that we are concerned here with simultaneous approximation of distinct functions on disjoint sets by a single polynomial. The approximations are measured only on the polygons $P_{j}$; nothing is required in the "don't care" space in-between. For digital filtering, the polygons would typically be intervals corresponding to pass and stop bands, and for matrix iterations, they would be regions approximately enclosing various components of the spectrum or pseudospectra of the matrix.

According to results of approximation theory going back to Chebyshev, there exists a polynomial $p_{n}$ that minimizes $(7)$, and it is unique $[2,3,30]$. What is interesting is how much about $p_{n}$ can be inferred from the Green's function. We summarize two of the known facts about this problem as follows:

THEOREM 2. Let $\left\{p_{n}\right\}$ and $\left\{E_{n}\right\}$ be the optimal polynomials and corresponding errors for the Polynomial Approximation Problem, let $g$ be the Green's function, and let the critical level curve and the asymptotic convergence factor $\rho$ be defined as in Section 4. Then

(a) $\lim \sup _{n \rightarrow \infty} E_{n}^{1 / n}=\rho$.

(b) ("Overconvergence") $p_{n}(z) \rightarrow h_{j}(z)$ as $n \rightarrow \infty$, not only for $z \in P_{j}$, but for all $z$ in the region enclosed by the component of the critical level curve enclosing $P_{j}$, with uniform convergence on compact subsets. Conversely, $p_{n}(z)$ does not converge uniformly to $h_{j}(z)$ in any neighborhood of any point on the critical level curve.

These results are due in important measure to Walsh, and are proved in his treatise [30]; see Theorems 4.5-4.7 and 4.11 and the discussions surrounding them. Some of this material was presented earlier in a 1934 paper by Walsh and Helen G. Russell [31], which attributes previous related work to Faber, Bernstein, M. Riesz, Fejér, and Szegö. The formulations as we have stated them are not very sharp. The original results of Walsh are more quantitative, and they were sharpened further by Fuchs, especially for the case in which $E$ is a collection of intervals [8,9].

Theorem 2 concerns the exact optimal polynomials for the Polynomial Approximation Problem, which are usually unknown and difficult to compute. Walsh showed that the same conclusions apply more generally, however, to any sequence of polynomials that is maximally convergent, which means, any sequence $\left\{p_{n}\right\}$ whose errors $\left\{E_{n}\right\}$ as defined by (7) satisfy condition (a) of Theorem 2. Now then, how can we 
construct maximally convergent sequences? Further results of Walsh establish that this can be done via interpolation in suitably distributed points:

THEOREM 3. Consider a sequence of sets of $n+1=1,2,3, \ldots$ points $\left\{\beta_{j}^{(n)}\right\}$ either lying in $E$ or converging uniformly to $E$ as $n \rightarrow \infty$, and suppose that the potential they generate in the sense of Section 4 converges uniformly to the Green's function $g(z)$ on all compact subsets disjoint from $E$. Let $\left\{p_{n}\right\}$ be the sequence of polynomials of degrees $n=0,1,2, \ldots$ generated by interpolation in these points of a function $h(z)$ defined in $\mathbf{C}$ with $h(z)=h_{j}(z)$ in a neighborhood of each $P_{j}$. This sequence of polynomials is maximally convergent for the Polynomial Approximation Problem.

THEOREM 4. The overconvergence result of Theorem 2(b) applies to any sequence $\left\{p_{n}\right\}$ of maximally convergent polynomials for the Polynomial Approximation Problem.

For proofs see Theorems 4.11 and 7.2 of [30] and the discussions nearby.

Theorem 3 implies that once the Green's function $g(z)$ is known, it can be used to construct maximally convergent polynomials by a variety of methods. The simplest approach is to take $p_{n}$ to be the polynomial defined by interpolation of $h_{j}$ in the pre-images along the boundary of $P_{j}$ of roots of unity in the $w$-plane:

$$
\begin{gathered}
z_{k}=\Phi^{-1}\left(e^{i \theta_{k}}\right), \\
\theta_{k}=\frac{\left(k-\frac{1}{2}\right) \pi}{n+1}, \quad k=1, \ldots, n+1 .
\end{gathered}
$$

Alternatively, and perhaps slightly more effective in practice, we may adjust the points along the boundary of each polygon $P_{j}$. Given $n$, we determine by (8) and (9) the number $n_{j}$ of interpolation points that will lie on the boundary of $P_{j}$. If $\underline{\theta}$ and $\bar{\theta}$ are the lower and upper edge angles along the unit circle in the $w$-plane corresponding to $P_{j}$ (in the notation of Fig. $1, \underline{\theta}=\tau_{j} / i$ and $\bar{\theta}=\sigma_{j} / i$ ), then we define the actual interpolation points along the boundary of $P_{j}$ by (8) and

$$
\theta_{k}=\underline{\theta}+\frac{\left(k-\frac{1}{2}\right)(\bar{\theta}-\underline{\theta})}{n_{j}}, \quad k=1, \ldots, n_{j} .
$$

Both of the choices (9) and (10) lead to maximal convergence as in Theorem 3.

Figure 8 illustrates the ideas of Theorems 2 and 3, especially the phenomenon of overconvergence. Here we continue with the same geometry as in Fig. 3 and construct near-best approximations $p_{n}(z)$ by interpolation of the constants -1 on the hexagon and +1 on the square in the points described by (8) and (9). These two constants represent distinct entire functions, so the polynomials $\left\{p_{n}(z)\right\}$ cannot converge globally. They converge on regions much larger than the polygons themselves, however, as the figure vividly demonstrates: all the way out to the critical "figure- 8 " level curve, in keeping with Theorem 2. The colors correspond to just the real part of $p_{n}(z)$, but the imaginary part (not shown) looks similar, taking values close to zero inside the figure- 8 and growing approximately exponentially outside.

Our final example, motivated by the work of Mitchell, Shen and Strang on digital filters, takes a special case in which $E$ consists of two real intervals. Consider the approximation problem defined by a "stop band" $P_{1}=[-1,-0.4]$ with $h_{1}(x)=0$ and a "pass band" $P_{2}=[-0.3,1]$ with $h_{2}(x)=1$. That is, the problem is to find polynomials $p_{n}$ of degree $n$ that minimize

$$
E_{n}=\max \left\{\max _{x \in[-1,-0.4]}|p(x)|, \max _{x \in[-0.3,1]}|p(x)-1|\right\} .
$$





[ The original image
is of higher quality,
and is attached at and is attached at

Figure 8. Illustration of the overconvergence phenomenon of Theorem 2(b) and Theorem 4. On the same two-polygon region as in Fig. 3, a polynomial $p(z)$ is sought that approximates the values -1 on the hexagon and +1 on the square. For this figure, $p$ is taken as the degree- 29 near-best approximation defined by interpolation in 30 pre-images of roots of unity in the unit circle under the conformal map $z=\Phi^{-1}(w)$ (eqs. (8) and (9)); a similar plot for the exactly optimal polynomial would not look much different. The figure shows $\operatorname{Re} p(z)$ by a blue-red color scale together with the polygons, the interpolation points, and the figure- 8 shaped critical level curve of the Green's function. Not just on the polygons themselves, but throughout the two lobes of the figure- $8, \operatorname{Re} p(z)$ comes close to the constant values -1 and +1 . Outside, it grows very fast.

Our Schwarz-Christoffel computations (elementary, since the more difficult first map $f_{1}$ of Fig. 1 is the identity in this case) show that the asymptotic convergence factor is $\rho=0.947963$, the capacity is $C=0.499287$, the critical point and level are $z_{c}=$ -0.350500 and $g_{c}=0.053440$, and the harmonic measures are $\mu_{1}=0.385927$ and $\mu_{2}=0.614073$.

For $n=19$, Fig. 9 plots the near-best polynomial $p_{n}$ defined by interpolation in the points defined by (8) and (10). The polynomial has approximately equiripple form, suggesting that it is close to optimal. The horizontal dashed lines suggest the error in this approximation, but it is clear they do not exactly touch the maximalerror points of the curve. In fact, these dashed lines are drawn at distances $\pm \rho^{n} / \sqrt{n}$ from the line to be approximated, where $\rho$ is the asymptotic convergence factor; the adjustment by $\sqrt{n}$ is suggested by the theorems of Fuchs [8]. In other words, these lines mark a predicted error based on the Green's function, not the actual error of the polynomial approximation obtained from it.

Figure 10 shows the actual optimal polynomial for this approximation problem, with equiripple behavior. Something looks wrong here-the errors seem bigger than 




Figure 9. The near-best polynomial $p_{19}(x)$ obtained from the Green's function by interpolation in the 20 points $(8),(10)$ of 0 in the stop band $[-1,-0.4]$ and 1 in the pass band $[-0.3,1]$. The polynomial is not optimal, but it is close.

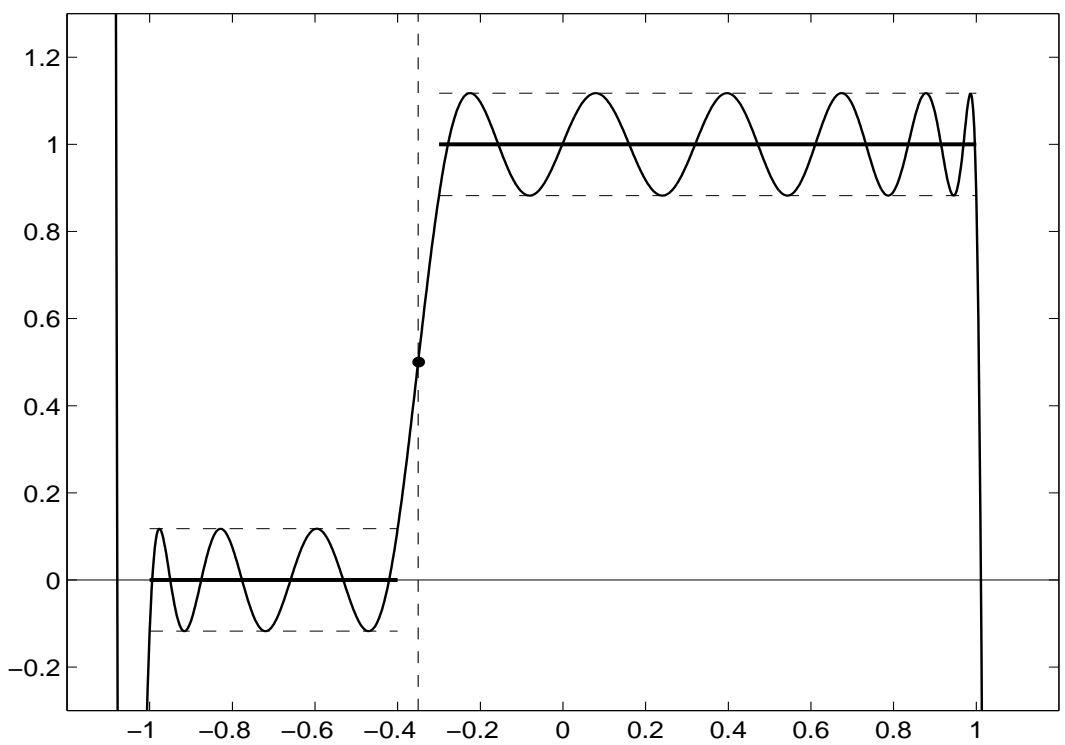

Figure 10. Same as Fig. 9, but for the optimal polynomial $p_{19}(x)$ computed by the Remes algorithm. At first glance, the approximation looks worse. In fact, it is better, since there are large errors in Fig. 9 at the inner edges of the stop and pass bands. 



Figure 11. Comparison of Green's function predictions (solid curves) with exact equiripple approximations (dots) for the example (11). Details in the text.

in Fig. 9, not smaller! In fact, Fig. 9 is not as good as it looks. At the right edge of the stop band and at the left edge of the pass band, for $x \approx-0.4$ and $x \approx-0.3$, there are large errors. The numerical results line up as follows:

Optimal error $E_{n}: 0.1176$

Error $\rho^{n} / \sqrt{n}$ estimated from Green's function: 0.0831

Error in polynomial obtained from Green's function: 0.2030

In some engineering applications, of course, Fig. 9 might represent a better filter than Fig. 10 after all.

Figure 11 presents three comparisons between properties of the exactly optimal polynomials $p_{n}(x)$ for this problem (solid dots) and predictions based on the Green's function (curves). Plot (a) compares the error $E_{n}$ with the prediction $\rho^{n} / \sqrt{n}$ (the distances between the horizontal dashed lines in Figs. 9 and 10). Evidently these quantities differ by a factor of less than 2. Plot (b) compares the proportion of the interpolation points that lie in the stop band with the harmonic measure $\mu_{1}$. The agreement is as good as one could hope for. Finally, plot (c) compares the point $x$ in $[-1,1]$ at which the optimal polynomial satisfies $p(x)=0.5$ (the vertical dashed line of Fig. 10) with the critical point $z_{c}$ (the vertical dashed line of Fig. 9). Evidently the Green's function makes a good prediction of this transition point for finite $n$ and exactly the right prediction as $n \rightarrow \infty$, as it must by Theorem 2 (b). 
7. Weighted Green's functions for weighted approximation. In signal processing applications, rather than a uniform approximation, one commonly wants an approximation corresponding to errors weighted by different constants $W_{j}$ in different intervals $P_{j}$. In closing we note that the techniques we have described can be generalized to this case by considering a weighted Green's function in which (1b) is replaced by the condition

$$
g(z) \rightarrow-n^{-1} \log W_{j} \quad \text { for } z \rightarrow P_{j},
$$

which depends on $n$. The function $g$ can now be determined by a conformal map onto a semi-infinite strip whose end is jagged, with the $K$ segments lying at real parts $-n^{-1} \log W_{j}$. Numerical experiments show that this method is effective, and very general theoretical developments along these lines are described in the treatise of Saff and Totik [21].

\section{REFERENCES}

[1] L. Ahlfors, Conformal Invariants: Topics in Geometric Function Theory, McGraw-Hill, New York, 1973.

[2] N. Akhiezer, Theory of Approximation, Ungar, New York, 1956.

[3] E. W. Cheney, Introduction to Approximation Theory, McGraw-Hill, New York, 1966.

[4] T. A. Driscoll, Algorithm 756: A MATLAB toolbox for Schwarz-Christoffel mapping, ACM Trans. Math. Software 22 (1996), 168-186. See also http://amath.colorado.edu/appm/fac ulty/tad/research/sc.html.

[5] T. A. Driscoll, K.-C. Toh, and L. N. Trefethen, From potential theory to matrix iterations in six steps, SIAM Review 40 (1998), 547-578.

[6] B. Fischer, Polynomial Based Iteration Methods for Symmetric Linear Systems, Wiley-Teubner, New York, 1996.

[7] W. H. J. Fuchs, Topics in the Theory of Functions of One Complex Variable, D. van Nostrand, Princeton, 1962.

[8] W. H. J. Fuchs, On the degree of Chebyshev approximation on sets with several components, Izv. Akad. Nauk. Armyan. SSR 13 (1978), 396-404.

[9] W. H. J. Fuchs, On Chebyshev approximation on several disjoint intervals, in B. Aupetit, ed., Complex Approximation, Birkhäuser, 1980, pp. 66-74.

[10] D. Gaier, Lectures on Complex Approximation, Birkhäuser, Boston, 1987.

[11] A. Greenbaum, Iterative Methods for Solving Linear Systems, SIAM, Philadelphia, 1997.

[12] P. Henrici, Applied and Computational Complex Analysis, v. 3, Wiley, New York, 1986.

[13] E. Hille, Analytic Function Theory, v. 2, Chelsea, New York, 1962.

[14] P. D. Lax and C. D. Levermore, The small dispersion limit of the Korteweg-de Vries equation. II, Commun. Pure Appl. Math. 36 (1983), 571-593.

[15] S. Mitchell, dissertation, Dept. of Elect. Engr., Cornell University, in preparation.

[16] A. V. Oppenheim and R. W. Schafer, Discrete-Time Signal Processing, Prentice-Hall, Englewood Cliffs, NJ, 1989.

[17] T. W. Parks and C. S. Burrus, Digital Filter Design, Wiley, New York, 1987.

[18] T. W. Parks and J. H. McClellan, Chebyshev approximation for nonrecursive digital filters with linear phase, IEEE Trans. Circuit Theory CT-19 (1972), 189-194.

[19] L. R. Rabiner and B. Gold, Theory and Applications of Digital Signal Processing, Prentice-Hall, Englewood Cliffs, NJ, 1975.

[20] Y. Saad, Iterative Methods for Sparse Linear Systems, PWS Publishing, Boston, 1996.

[21] E. B. Saff and V. Totik, Logarithmic Potentials with External Fields, Springer, Berlin, 1997.

[22] J. Shen and G. Strang, The asymptotics of optimal (equiripple) filters, IEEE Trans. Signal Proc., to appear.

[23] J. Shen, G. Strang, and A. Wathen, The potential theory of several intervals and its applications, Applied Math. and Opt., submitted.

[24] E. B. Stahl and V. Totik, General Orthogonal Polynomials, Cambridge U. Press, Cambridge, 1992. 
[25] G. Szegő, Bermerkungen zu einer Arbeit von Herrn M. Fekete: Über die Verteilung der Wurzeln bei gewissen algebraischen Gleichungen mit ganzzahligen Koeffizienten, Math. Zeit. 21 (1924) 203-208.

[26] L. N. Trefethen, Numerical computation of the Schwarz-Christoffel transformation, SIAM J. Sci. Stat. Comput 1 (1980), 82-102.

[27] L. N. Trefethen, Analysis and design of polygonal resistors by conformal mapping, J. Appl. Math. Phys. 35 (1984), 692-704.

[28] L. N. Trefethen and D. Bau, III, Numerical Linear Algebra, SIAM, Philadelphia, 1997.

[29] M. Tsuji, Potential Theory in Modern Function Theory, Dover, New York, 1959.

[30] J. L. Walsh, Interpolation and Approximation by Rational Functions in the Complex Domain, 5th ed., Amer. Math. Soc., Providence, RI, 1969.

[31] J. L. Walsh and H. G. Russell, On the convergence and overconvergence of sequences of polynomials of best simultaneous approximation to several functions analytic in distinct regions, Trans. Amer. Math. Soc. 36 (1934), 13-28.

[32] H. Widom, Extremal polynomials associated with a system of curves in the complex plane, Adv. Math. 3 (1969), 127-232. 